\title{
AN INTEGRAL ANALOGUE OF LAPLACE'S EQUATION ${ }^{1}$
}

\author{
MAXWELL READE AND E. F. BECKENBACH
}

1. Introduction. A general problem of a formal nature may be stated, for functions of two variables, as follows. Let a class of functions $x(u, v)$, or a class of sets of functions $x_{j}(u, v), j=1,2, \cdots, n$, defined in a finite simply connected domain $D$, be characterized as satisfying a set of one or more differential equations,

$$
A=0 .
$$

If in (1) we replace the partial derivatives

$$
\frac{\partial y}{\partial u}, \quad \frac{\partial y}{\partial v}
$$

by the line integrals

$$
\frac{1}{\pi r^{2}} \int_{C_{r}} y d v, \quad-\frac{1}{\pi r^{2}} \int_{C_{r}} y d u,
$$

respectively, where $C_{r}$ is an arbitrary circle, of variable radius $r$, lying in $D$, we obtain a set of integral equations,

$$
B=0 \text {, }
$$

analogous to (1). Our problem is the determination of the class of functions, or of sets of functions, characterized by (2).

If $y(u, v)$ has continuous first partial derivatives, then

$\frac{1}{\pi r^{2}} \int_{C_{r}} y d v=y_{u}\left(u_{0}, v_{0}\right)+o\left(r^{0}\right), \quad-\frac{1}{\pi r^{2}} \int_{C_{r}} y d u=y_{v}\left(u_{0}, v_{0}\right)+o\left(r^{0}\right)$,

where the subscripts $u, v$ denote partial differentiation, where $r$ and $\left(u_{0}, v_{0}\right)$ are the length of the radius and the coordinates of the center of $C_{r}$, respectively, and where $o\left(r^{\alpha}\right)$ denotes a quantity (not always the same quantity) such that

$$
\lim _{r \rightarrow 0} \frac{o\left(r^{\alpha}\right)}{r^{\alpha}}=0 .
$$

Hence, for functions for which the derivatives involved in (1) are continuous, (1) is equivalent to

$$
B=o\left(r^{0}\right),
$$

${ }^{1}$ Presented in part to the Society, June 20, 1940, by E. F. Beckenbach. 
and a necessary condition that (2) hold for all circles in $D$ is that (1) hold for all points in $D$; we might consider (1) to be a special case of (2), corresponding to $r=0$.

Further local differential conditions, usually more restrictive than (1), but less severe than the integral condition (2), are obtained by replacing (2) by

$$
B=o\left(r^{\alpha}\right)
$$

for significant values of the exponent $\alpha$. On the other hand, even more restrictive than (2) is

$$
B^{*}=0 \text {, }
$$

where $B^{*}$ is obtained from $B$ by replacing

$$
\frac{1}{\pi r^{2}} \int_{C_{r}} \text { by } \int_{\gamma} \text {, }
$$

where $\gamma$ is an arbitrary closed rectifiable Jordan curve in $D$.

For example, if (1) is

$$
x_{1, v}=x_{2, u},
$$

the corresponding integral equation (4) is the familiar

$$
\int_{\gamma} x_{1} d u+x_{2} d v=0
$$

Or if (1) is the set of Cauchy-Riemann differential equations,

$$
x_{1, u}=x_{2, v}, \quad x_{1, v}=-x_{2, u},
$$

the corresponding integral equations (4) are

$$
\int_{\gamma} x_{1} d v=-\int_{\gamma} x_{2} d u, \quad \int_{\gamma} x_{1} d u=\int_{\gamma} x_{2} d v,
$$

which can be written together as the Cauchy integral equation

$$
\int_{\gamma} f(z) d z \equiv \int_{\gamma}\left[x_{1}(u, v)+i x_{2}(u, v)\right](d u+i d v)=0 .
$$

Again, analogous to the differential conditions

$$
\sum_{j=1}^{3} x_{j, u}^{2}=\sum_{j=1}^{3} x_{j, v}^{2}, \quad \sum_{j=1}^{3} x_{j, u} x_{j, v}=0,
$$

that is, $E=G$ and $F=0$, which characterize conformal, or isothermic, 
maps, we have considered ${ }^{2}$ the integral conditions

$$
\begin{aligned}
& \sum_{j=1}^{3}\left[\int x_{j}(u, v) d u\right]^{2}=\sum_{j=1}^{3}\left[\int x_{j}(u, v) d v\right]^{2}, \\
& \sum_{j=1}^{3}\left[\int x_{j}(u, v) d u\right]\left[\int x_{j}(u, v) d v\right]=0 .
\end{aligned}
$$

The hierarchy of integral conditions discussed above leads this time to an integral characterization of isothermic maps; of isothermic spherical maps, both those which carry circles into circles and those which do not; of isothermic maps on minimal surfaces; and of isothermic plane maps. Moreover, the investigation has yielded ${ }^{3}$ unex- $^{-}$ pected information concerning mean-value surfaces.

2. Characterization of harmonic functions. In this paper we shall apply the above method to set up an integral equation analogous to Laplace's second order differential equation,

$$
\Delta x \equiv \frac{\partial^{2} x}{\partial u^{2}}+\frac{\partial^{2} x}{\partial v^{2}}=0,
$$

which characterizes harmonic functions. This will illustrate how the method may be applied to equations of order higher than the first: we shall establish a second order integral equation characterizing harmonic functions. Our integral equation is not to be confused with other integral equations characterizing harmonic functions,

$$
\begin{gathered}
\int_{\gamma} \frac{d x}{d n} d s=0, \\
x\left(u_{0}, v_{0}\right)=\frac{1}{2 \pi} \int_{0}^{2 \pi} x\left(u_{0}+r \cos \phi, v_{0}+r \sin \phi\right) d \phi, \\
x\left(u_{0}, v_{0}\right)=\frac{1}{\pi r^{2}} \iint_{s^{2}+t^{2} \leqq r^{2}} x\left(u_{0}+s, v_{0}+t\right) d s d t
\end{gathered}
$$

rather, it seems to be more nearly analogous to the (first order) Cauchy integral equation (5) characterizing analytic functions of a complex variable. As in the case of the Cauchy integral equation,

\footnotetext{
2 Maxwell Reade and E. F. Beckenbach, Generalizations to space of the Cauchy and Morera theorems, Proceedings of the National Academy of Sciences, vol. 25 (1939), pp. 92-97; also, Transactions of this Society, vol. 49 (1941), pp. 354-377.

${ }^{3}$ Maxwell Reade and E. F. Beckenbach, Mean-value surfaces, to appear in an early issue of this Bulletin.
} 
there is here no hierarchy of results: a weak condition of type (3) implies that $x(u, v)$ is harmonic, while the condition that $x(u, v)$ is harmonic implies a strong result of type (4).

We note that

$$
\Delta x \equiv \bar{\lambda}(\lambda x) \equiv \lambda(\bar{\lambda} x)
$$

where

$$
\lambda \equiv \frac{\partial}{\partial u}+i \frac{\partial}{\partial v}, \quad \bar{\lambda} \equiv \frac{\partial}{\partial u}-i \frac{\partial}{\partial v}
$$

Let

$$
\begin{array}{lll}
C: \quad u=s(\tau), & v=t(\tau), & 0 \leqq \tau \leqq 1 ; \\
C^{*}: \quad u=\xi(\mu), & v=\eta(\mu), & 0 \leqq \mu \leqq 1,
\end{array}
$$

be arbitrary closed rectifiable Jordan curves in the $u, v$-plane such that for each $\tau_{0}, 0 \leqq \tau_{0} \leqq 1$, the curve

$$
C\left[s\left(\tau_{0}\right), t\left(\tau_{0}\right)\right]: \quad u=s\left(\tau_{0}\right)+\xi(\mu), \quad v=t\left(\tau_{0}\right)+\eta(\mu), \quad 0 \leqq \mu \leqq 1,
$$

lies in $D$. Making the prescribed replacements in (6), in accordance with (7), we find the following integral analogues of Laplace's differential equation:

$$
\begin{aligned}
& \int_{C}\left[\int_{C(s, t)} x(s+\xi, t+\eta)(d \xi-i d \eta)\right](d s+i d t)=0, \\
& \int_{C}\left[\int_{C(s, t)} x(s+\xi, t+\eta)(d \xi+i d \eta)\right](d s-i d t)=0 .
\end{aligned}
$$

Now both (8) and (9) turn out to characterize harmonic functions; since the analysis is the same in the two cases, we shall confine our attention to just one of them, namely (8).

If (8) holds for fixed closed rectifiable Jordan curves $C$ and $C^{*}$, then we shall say that (8) holds for the curve-pair $\left(C, C^{*}\right)$.

THEOREM 1. If the real function $x(u, v)$ is continuous in a finite simply connected domain $D$, then a necessary and sufficient condition that $x(u, v)$ be harmonic in $D$ is that

$$
\int_{C}\left[\int_{C(s, t)} x(s+\xi, t+\eta)(d \xi-i d \eta)\right](d s+i d t)=0
$$

hold for all curve-pairs $\left(C, C^{*}\right)$ for which the curves $C(s, t)$ all lie in $D$.

Necessity. If $x(u, v)$ is harmonic in $D$, and if $\xi(\mu)$ and $\eta(\mu)$ are the 
coordinate functions of an arbitrary fixed closed rectifiable Jordan curve $C^{*}$, then

$$
\int_{C(s, t)} x(s+\xi, t+\eta)(d \xi-i d \eta)
$$

where $\{C(s, t)\}$ is the family of curves

$$
C(s, t): \quad u=s+\xi(\mu), \quad v=t+\eta(\mu), \quad 0 \leqq \mu \leqq 1,
$$

defines a function $F(w)$ of the complex variable $w=s+i t ; F(w)$ is defined on a point-set $D\left(C^{*}\right)$ of the $u$, v-plane, consisting of finite open simply connected pieces. By Green's lemma, it follows that

$$
F(w)=-\iint_{D(s, t)}\left[x_{2}(s+\xi, t+\eta)+i x_{1}(s+\xi, t+\eta)\right] d \xi d \eta
$$

where $D(s, t)$ denotes the interior of the curve $C(s, t)$, and where $x_{1}(u, v) \equiv \partial x / \partial u, x_{2}(u, v) \equiv \partial x / \partial v$. By Leibnitz' rule, we have

$$
\begin{aligned}
\frac{\partial}{\partial s} \iint_{D(s, t)} x_{2}(s+\xi, t+\eta) d \xi d \eta & =\iint_{D(s, t)} x_{1,2}(s+\xi, t+\eta) d \xi d \eta \\
& =\frac{\partial}{\partial t} \iint_{D(s, t)} x_{1}(s+\xi, t+\eta) d \xi d \eta
\end{aligned}
$$

(13) $\frac{\partial}{\partial t} \iint_{D(s, t)} x_{2}(s+\xi, t+\eta) d \xi d \eta=\iint_{D(s, t)} x_{2,2}(s+\xi, t+\eta) d \xi d \eta$,

(14) $\frac{\partial}{\partial s} \iint_{D(s, t)} x_{1}(s+\xi, t+\eta) d \xi d \eta=\iint_{D(s, t)} x_{1,1}(s+\xi, t+\eta) d \xi d \eta$.

Since $x(u, v)$ is harmonic in $D,(13)$ and (14) yield

(15) $\frac{\partial}{\partial t} \iint_{D(s, t)} x_{2}(s+\xi, t+\eta) d \xi d \eta=-\frac{\partial}{\partial s} \iint_{D(s, t)} x_{1}(s+\xi, t+\eta) d \xi d \eta$.

From (11), (12) and (15) it follows that $F(w)$ is analytic in $D\left(C^{*}\right)$. Since for each closed rectifiable Jordan curve $C$ lying in $D\left(C^{*}\right)$ the curve $C$ encloses only points belonging to $D\left(C^{*}\right)$, it follows from Cauchy's theorem that $(10)$ holds for $\left(C, C^{*}\right)$. Since $C^{*}$ is an arbitrary closed rectifiable Jordan curve, it follows that (10) holds for all curve-pairs $\left(C, C^{*}\right)$ for which the curves $C(s, t)$ all lie in $D$.

Sufficiency. If $x(u, v)$ is continuous in $D$, then for a fixed positive $\rho$, the averaging function ${ }^{4}$

${ }^{4}$ For a list of properties of averaging functions, see H. E. Bray, Proof of a formula for an area, this Bulletin, vol. 29 (1923), pp. 264-270. 


$$
x(u, v ; \rho) \equiv \frac{1}{\pi \rho^{2}} \iint_{\xi^{2}+\eta^{2} \leqq \rho^{2}} x(u+\xi, v+\eta) d \xi d \eta
$$

has continuous partial derivatives of the first order; of course, $x(u, v ; \rho)$ can be defined thus only for an open subset $D_{\rho}$ of $D$, but this is of no consequence since $\rho$ is arbitrarily small. We have the following equation

$$
\bar{\lambda} x(u, v ; \rho)=\frac{i}{\pi \rho^{2}} \int_{\xi^{2}+\eta^{2}=\rho^{2}} x(u+\xi, v+\eta)(d \xi-i d \eta) .
$$

If (10) holds, then it follows from (16) and the theorem of Morera that $\bar{\lambda} x(u, v ; \rho)$ is analytic in $D_{\rho}$, and hence it follows from the CauchyRiemann equations that $x(u, v ; \rho)$ is harmonic in $D_{\rho}$. Since in any closed subset of $D, x(u, v ; \rho) \rightarrow \vec{\rightarrow} x(u, v)$ as $\rho \rightarrow 0$, it follows that $x(u, v)$ is harmonic in $D$.

3. Lessening the sufficiency conditions. Actually, we need a good deal less to prove $x(u, v)$ harmonic in $D$. In the first place, letting $\Im \phi$ denote the imaginary part of the complex function $\phi$, we have the following result.

THEOREM 2. If the real function $x(u, v)$ is continuous in a finite simply connected domain. $D$, then

$$
J \int_{C}\left[\int_{C(s, t)} x(s+\xi, t+\eta)(d \xi-i d \eta)\right](d s+i d t)=0
$$

holds for all curve-pairs $\left(C, C^{*}\right)$ for which the curves $C(s, t)$ all lie in $D$.

Proof. The repeated average,

$$
x\left(u, v ; \rho^{(2)}\right) \equiv \frac{1}{\pi \rho^{2}} \iint_{\beta^{2}+\zeta^{2} \leqq \rho^{2}} x(u+\beta, v+\zeta ; \rho) d \beta d \zeta,
$$

has continuous partial derivatives of the second order. We have

$$
\begin{aligned}
J \int_{C}\left[\int_{C(s, t)} x(s+\xi, t\right. & \left.\left.+\eta ; \rho^{(2)}\right)(d \xi-i d \eta)\right](d s+i d t) \\
= & \int_{C}\left[\int_{C(s, t)} x\left(s+\xi, t+\eta ; \rho^{(2)}\right) d \xi\right] d t \\
& -\int_{C}\left[\int_{C(s, t)} x\left(s+\xi, t+\eta ; \rho^{(2)}\right) d \eta\right] d s
\end{aligned}
$$


hence, by Green's lemma and Leibnitz' rule, we obtain

$$
\begin{gathered}
J \int_{C}\left[\int_{C(s, t)} x\left(s+\xi, t+\eta ; \rho^{(2)}\right)(d \xi-i d \eta)\right](d s+i d t) \\
=\iint_{D(C)}\left\{\int \int _ { D ( s , t ) } \left[-\frac{\partial^{2} x\left(s+\xi, t+\eta ; \rho^{(2)}\right)}{\partial s \partial \eta}\right.\right. \\
\left.\left.+\frac{\partial^{2} x\left(s+\xi, t+\eta ; \rho^{(2)}\right)}{\partial t \partial \xi}\right] d \xi d \eta\right\} d s d t
\end{gathered}
$$

where $D(C)$ denotes the interior of $C$ and where $D(s, t)$ denotes the interior of $C(s, t)$. From (18) it follows that

$$
J \int_{C}\left[\int_{C(s, t)} x\left(s+\xi, t+\eta ; \rho^{(2)}\right)(d \xi-i d \eta)\right](d s+i d t)=0
$$

holds for all curve-pairs $\left(C, C^{*}\right)$ for which the curves $C(s, t)$ all lie in the region of definition of $x\left(u, v ; \rho^{(2)}\right)$. Since in any closed subset of $D, x\left(u, v ; \rho^{(2)}\right) \rightarrow x(u, v)$ as $\rho \rightarrow 0$, we obtain (17) from (19).

It follows that harmonic functions are characterized by the vanishing of only the real part of the left-hand member of (10).

In the second place, we can replace the strong integral condition (10), which is of type (4), by a weaker point condition of type (3). Let $k$ be a fixed positive constant, let $C\left(u_{0}, v_{0} ; r\right)$ denote an arbitrary circle in $D$ with center at $\left(u_{0}, v_{0}\right)$ and of radius $r$, and let $C\left(u_{0}+s, v_{0}+t ; k r\right)$ denote the circles in $D$ having common radii $k r$ and having their centers on the circumference of the circle $C\left(u_{0}, v_{0} ; r\right)$. If $R \phi$ denotes the real part of the complex function $\phi$, then we have the following result.

THEOREM 3. If the real function $x(u, v)$ is continuous in a finite simply connected domain $D$, then a necessary and sufficient condition that $x(u, v)$ be harmonic in $D$ is that for each point $\left(u_{0}, v_{0}\right)$ in $D$,

$$
\begin{array}{r}
R \int_{C\left(u_{0}, v_{0} ; r\right)}\left[\int_{C\left(u_{0}+s, v_{0}+t ; k r\right)} x\left(u_{0}+s+\xi, v_{0}+t+\eta\right)\right. \\
\cdot(d \xi-i d \eta)](d s+i d t)=o\left(r^{4}\right),
\end{array}
$$

the integrals being taken once around the circles in the positive (counterclockwise) sense.

Proof. If it is given that (20) holds, then also 


$$
\begin{array}{r}
R \int_{C\left(u_{0}, v_{0} ; r\right)}\left[\int_{C\left(u_{0}+s, v_{0}+t ; k r\right)} x\left(u_{0}+s+\xi, v_{0}+t+\eta ; \rho^{(2)}\right)\right. \\
\cdot(d \xi-i d \eta)](d s+i d t)=o\left(r^{4}\right) .
\end{array}
$$

A computation yields

$$
\begin{aligned}
& R \int_{C\left(u_{0}, v_{0} ; r\right)} {\left[\int_{C\left(u_{0}+s, v_{0}+t ; k r\right)} x\left(u_{0}+s+\xi, v_{0}+t+\eta ; \rho^{(2)}\right)\right.} \\
&\cdot(d \xi-i d \eta)](d s+i d t)=\pi^{2} k^{2} r^{4} \Delta x\left(u_{0}, v_{0} ; \rho^{(2)}\right)+o\left(r^{4}\right) .
\end{aligned}
$$

By (21) and (22), $x\left(u, v ; \rho^{(2)}\right)$ is harmonic in its region of definition, whence, as above, $x(u, v)$ is harmonic in $D$.

If, conversely, it is given that $x(u, v)$ is harmonic, then we obtain (20) from an expansion similar to (22) for the function $x(u, v)$.

We note that an alternate proof of Theorem 1 might be given along the line of the above proof of Theorem 2, and that the proof here given of Theorem 3 contains still another proof of the sufficiency part of Theorem 1.

Ohio State University and

The University OF MichigaN 